\title{
La ruta bioceánica y las políticas públicas en los municipios fronterizos de Porto Murtinho/Brasil - Carmelo Peralta/Paraguay
}

\author{
The bioceanic route and public policies of the border cities of Porto Murtinho/ \\ Brazil - Carmelo Peralta/Paraguay \\ A rota bioceânica e políticas públicas dos municípios fronteiriços de Porto \\ Murtinho/Brasil - Carmelo Peralta/Paraguai
}

\author{
Luciane Pinho de Almeida ${ }^{1}$ \\ Gabriela Pereira da Silva ${ }^{2}$ \\ Gabriel de Souza Sales ${ }^{3}$ \\ Lívia Elena Cunha de Laura ${ }^{4}$
}

Recebido em 29/06/2021; revisado e aprovado em 17/09/2021; aceito em 13/10/2021

DOI: http://dx.doi.org/10.20435/inter.v22i4.3420

\begin{abstract}
Resumen: Con la llegada de la Ruta Bioceánica en el límite de los municipios de Porto Murtinho (Brasil) y Carmelo Peralta (Paraguay), la previsión es que se produzcan cambios sociales en la estructura de los municipios y su vida cotidiana. Este artículo pretende presentar reflexiones sobre los temas que permean las políticas públicas en la frontera, específicamente los municipios de Porto Murtinho y Carmelo Peralta. Este trabajo es el resultado del desarrollo de estudios para la elaboración del Plan Director para la ciudad de Porto Murtinho, los datos fueron recolectados entre septiembre de 2020 y junio de 2021 a través de entrevistas con los administradores públicos del municipio, cuestionarios aplicados con la población local, como así como también involucró investigaciones bibliográficas y documentales que ayuden a orientar nuevos caminos para las políticas locales. De los datos recogidos se observa que actualmente el municipio de Porto Murtinho cuenta con instalaciones públicas capaces de atender a la población, considerando su tamaño. Sin embargo, ante el aumento del flujo de personas y el posible aumento esperado de su población, las políticas públicas deben estar en consonancia con dicho desarrollo. Además, en la ciudad de Carmelo Peralta, la precariedad de los servicios públicos es evidente, especialmente en salud y asistencia social. Actualmente, muchos paraguayos ya utilizan servicios en el lado brasileño de la frontera porque no pueden acceder a su lugar de origen. Por ello, es de suma importancia que las políticas públicas consideren las particularidades de un municipio fronterizo que está por atravesar un momento de gran desarrollo, exigiendo diálogo y acuerdos bilaterales que tengan como objetivo garantizar los derechos sociales de las poblaciones locales.
\end{abstract}

Palabras clave: Frontera. Políticas Públicas. Ruta Bioceánica.

Abstract: With the arrival of the Bioceanic Route on the border of the municipalities of Porto Murtinho (Brazil) and Carmelo Peralta (Paraguay), the forecast is that social changes will take place regarding the structure of the municipalities and their daily lives. This article aims to present reflections on the issues that permeate public policies on the border, specifically the municipalities of Porto Murtinho and Carmelo Peralta. This work is the result of the development of studies for the preparation of the Master Plan for the city of Porto Murtinho, data were collected between September 2020 and June 2021 through interviews with public managers of the municipality, questionnaires applied with the local population, as well. as it involved bibliographic and documentary research that help guide new paths for local policies. From the data collected, it is observed that currently the municipality of Porto Murtinho has public facilities capable of serving the population, considering its size. However, in view of the increase in the flow of people and the possible expected increase in its population, public policies must be in line with such development. In addition, in the city of Carmelo Peralta, the precariousness of public services is evident, especially in health and social assistance. Currently, many Paraguayans already use services on the Brazilian side of the border because they cannot access their place of origin. Thus, it is extremely important that public policies consider the particularities of a border municipality that is about to go through a moment of great development, demanding dialogue and bilateral

\footnotetext{
1 Universidade Católica Dom Bosco, Campo Grande, Mato Grosso do Sul, Brasil. 2 Universidade Católica Dom Bosco, Campo Grande, Mato Grosso do Sul, Brasil. 3 Universidade Católica Dom Bosco, Campo Grande, Mato Grosso do Sul, Brasil. 4Universidade Católica Dom Bosco, Campo Grande, Mato Grosso do Sul, Brasil.
} 
agreements that aim to guarantee the social rights of local populations.

Key-words: Border. Public Policies. Bioceanic Route.

Resumo: Com a chegada da Rota Bioceânica na fronteira dos municípios de Porto Murtinho (Brasil) e Carmelo Peralta (Paraguai), a previsão é que ocorram transformações sociais no que tange à estrutura dos municípios e seu cotidiano. O presente artigo elege como objetivo apresentar reflexões sobre as questões que permeiam as políticas públicas na fronteira, especificamente nos municípios de Porto Murtinho e Carmelo Peralta. Este trabalho é resultado do desenvolvimento de estudos para a elaboração do Plano Diretor da cidade de Porto Murtinho. Os dados foram levantados entre setembro de 2020 e junho de 2021 por meio de entrevistas com gestores públicos do município, questionários aplicados com a população local, assim como envolveu pesquisas bibliográficas e documentais que auxiliem a direção de novos caminhos para as políticas locais. A partir das informações recolhidas, observa-se que atualmente o município de Porto Murtinho possui equipamentos públicos capazes de atender à população, considerando seu porte. No entanto, tendo em vista o aumento de fluxo de pessoas e possível incremento da população, as políticas públicas devem estar de acordo com tal desenvolvimento. Além disso, na cidade de Carmelo Peralta, evidencia-se a precariedade dos serviços públicos, especialmente de saúde e assistência social. Atualmente muitos paraguaios já utilizam serviços do lado brasileiro da fronteira por não conseguirem acesso em seu local de origem. Deste modo, é de suma importância que as políticas públicas considerem as particularidades de um município de fronteira que está prestes a passar por um momento de grande desenvolvimento, demandando diálogo e acordos bilaterais que tenham como objetivo a garantia dos direitos sociais das populações locais.

Palavras-chaves: Fronteira. Políticas Públicas. Rota Bioceânica.

\section{INTRODUCCIÓN}

Comprender las políticas públicas en municipios de frontera, como el de Porto Murtinho/ Brasil y el de Carmelo Peralta/Paraguay, implica antes de nada comprender las transformaciones sociales que ocurren en la rutina de la sociedad en diversos niveles en cuanto a su impacto y visibilidad por la población en general, desde pequeñas modificaciones hasta aquellas que resultan en un cambio de la estructura y organización de la sociedad. En este sentido, las transformaciones sociales son una respuesta de la sociedad frente a las condiciones de los tiempos presentes desarrolladas por el contexto histórico-social, implicando en los diversos factores que la calan, como el crecimiento económico, político, social entre otros, así como también desencadena diversos conflictos.

Es en el sentido de transformaciones sociales ocasionadas por el desarrollo de la actividad económica que la ciudad de Porto Murtinho, ubicada en la frontera de Mato Grosso del Sur con Paraguay, recibirá el paso de la Ruta Bioceánica, que pasará por cuatro países sudamericanos, Brasil, Paraguay, Argentina y Chile, reforzando las relaciones económicas entre los países, fortaleciendo el turismo local y posibilitando el desarrollo de las regiones, principalmente como vía de acceso para la exportación originaria del Centro-Oeste brasileño. De este modo, se cree que la Ruta Bioceánica propiciará cambios significativos para todos los territorios por los que pasará y no solo en la región del territorio de la frontera Brasil X Paraguay, ya que las cuestiones que afectan la Ruta Bioceánica tienen como principal objetivo expandir el desarrollo regional, posibilitando alternativas de transacciones comerciales más allá de las fronteras.

Se cuestiona, en ese sentido, cuáles serían los impactos sociales y cómo las políticas públicas deben estructurarse en el sentido de garantizar los derechos sociales a su población, lo que, por cierto, se vuelve pertinente en la medida en que crecen y se modifican las posibilidades dadas a la población.

El objetivo de este artículo es el de plantear reflexiones sobre las cuestiones que afectan a las políticas públicas en la frontera, específicamente en lo que respecta a los municipios de Porto 
Murtinho y Carmelo Peralta. Estas reflexiones son el resultado de los trabajos realizados en el desarrollo de los estudios para la construcción del plan director de la ciudad de Porto Murtinho/ MS, en el que fue también analizada la región de frontera y los impactos que la Ruta Bioceánica producirá en toda la región.

Los datos aquí discutidos se constituyen como el resultado de un estudio realizado entre septiembre de 2020 y junio de 2021 a través de investigaciones realizadas con la población de Porto Murtinho y de la frontera, con gestores públicos de la ciudad de Porto Murtinho, además de investigaciones documentales y bibliográficas que nortean posibles caminos para las políticas públicas regionales, principalmente en lo que afecta a los acuerdos bilaterales entre los países, teniendo como norte los derechos sociales y humanos de las poblaciones locales.

\section{LA RUTA BIOCEÁNICA Y LA FRONTERA BRASIL X PARAGUAY}

La Ruta Bioceánica trata de crear un corredor por carretera que conectará los territorios de cuatro países (Brasil, Paraguay, Argentina y Chile), con los objetivos de reducir el tiempo de desplazamiento de la carga, mejorar la logística del transporte y elevar la competitividad de las exportaciones a Asia, así como fomentar el turismo, estimular la creación de nuevos flujos de comercio regional y profundizar en la integración entre estos países. El Estado de Mato Grosso del Sur siempre idealizó una salida hacia el Pacífico y, por intermedio de los Gobiernos Municipal, Estatal y Federal, viene apoyando la realización de acciones concretas para su materialización. Esta realidad puede ser constatada por el asfaltado de la carretera que cruza el Chaco paraguayo, ya en su proceso de finalización, así como por la proyección de cuatro nuevos puertos fluviales en la región fronteriza, además de la licitación de un puente que ligará los municipios de Carmelo Peralta y Porto Murtinho.

El 21 de diciembre de 2015, los presidentes de Brasil, Argentina, Chile y Paraguay aprobaron la Declaración de Asunción, por medio de la cual fue creado un Grupo de Trabajo (GT) con la intención de realizar estudios técnicos y emprender acciones que hagan viable el Corredor por Carretera Bioceánico Porto Murtinho- Puertos del Norte de Chile. El referido GT está coordinado por los Ministerios de Asuntos Exteriores de los cuatro países e integrado por cinco grupos de trabajo que promueven la articulación de acciones diversas en aras de los gobiernos locales, sector privado, gobiernos centrales y universidades.

El Corredor por Carretera creará una importante conexión vial entre el Centro-Oeste brasileño y el Océano Pacífico. Tendrá su inicio en Mato Grosso del Sur (Brasil), en la ciudad de Porto Murtinho; cruzará el territorio paraguayo por Carmelo Peralta, Mariscal Estigarribia y Pozo Hondo (Paraguay); atravesará por el territorio argentino las ciudades de Misión La Paz, Tartagal, Jujuy y Salta (Argentina); ingresando en Chile por el Paso de Jama, hasta alcanzar los puertos de Antofagasta, Mejillones e Iquique, conforme la Figura 1. 
Figura 01 - Ruta Bioceánica Porto Murtinho-Puertos Norte de Chile

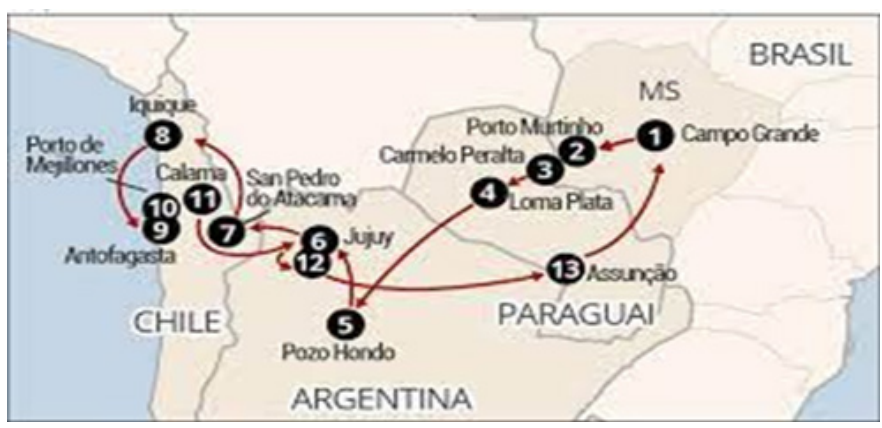

Fuente: Viegas, 2017 apud Ferreira, Castilho y Oliveira (2019)

Desde la perspectiva de Mato Grosso del Sur, el Corredor por Carretera Bioceánico le dará al agronegocio una salida al Océano Pacífico, permitiendo tanto la salida de la producción como la importación directa de insumos a precios más competitivos. De este modo, Porto Murtinho pasará a ser la puerta de entrada hacia el Corredor Bioceánico interconectando los países y favoreciendo la integración entre Brasil, Paraguay, Argentina y Chile.

Esta iniciativa de integración entre los países demuestra antes que nada una nueva vertiente de posicionamiento y expansión del territorio latinoamericano, y tiene fundamentos para el desplazamiento de las regiones más desarrolladas de estos países hacia áreas poco explotadas y en el fomento de la regionalización. De este modo, lo que estamos presenciando es el avance del desarrollo económico con la generación de focos de integración. Se puede apuntar, por lo tanto, que este no es un movimiento de un solo gobierno o específicamente de los gobiernos locales involucrados sino de todo un movimiento del propio capital que ahora se interioriza, buscando el desarrollo y la circulación de mercancías por regiones antes poco explotadas pero que poseen potencial de expansión y de generación de riqueza.

A partir de estas breves consideraciones sobre el Corredor Bioceánico Porto MurtinhoPuertos del Norte de Chile, se entiende la importancia de comprenderse la región de frontera y los municipios que destacamos aquí para esta discusión.

Las regiones de frontera poseen configuraciones diferenciadas y singulares, pues expresan formas culturales singulares constituidas por sujetos que se construyen mutuamente en un tiempo único, territorios que se arreglan a través de sujetos sociales en movimiento (KLEINSCHMITT; AZEVEDO; CARDIN, 2013), el espacio fronterizo es un viene-a-ser. Es decir, las fronteras son espacios dinámicos, fenómenos sociales plurales y en constante formación y transformación, locales en los que hay un entrelazado de los pueblos en el va y viene de brasileños e inmigrantes.

Souza (2009) plantea la siguiente reflexión sobre la relación entre los pueblos ubicados en la frontera:

Son espacios en los cuales lo local y lo internacional se articulan, estableciendo vínculos y dinámicas propias, construidas y reforzadas por los pueblos fronterizos. En ellos están presentes las identidades y las culturas nacionales de cada uno de los países involucrados, que construyen, reelaboran y constituyen otra cultura e identidad diferenciada, capaz de recrear un nuevo lugar, con aspectos regionales. Son regiones que no "respetan" las barreras existentes, ya que hay acción e interacción de los agentes fronterizos, estimulando dinámicas fronterizas informales. (SOUZA, 2009, pp. 106-107) 
Las regiones fronterizas, por lo tanto, se constituyen en la relación con el otro, con otras culturas y otras identidades, que rebasan los límites formales de cada país y se forman en una dinámica propia.

El territorio del municipio de Porto Murtinho está delimitado por una región fronteriza, constituida por dos países, Brasil y Paraguay, destacando las ciudades de Porto Murtinho-MS, del lado brasileño, y Carmelo Peralta, del lado paraguayo, con características semejantes y que constituyen relaciones constantes. Las demandas sociales ya existentes en la localidad serían más evidentes con el mayor flujo de personas derivadas del paso del Corredor Bioceánico, además de otros que puedan surgir en ese proceso.

Figura 02 - Porto Murtinho-Carmelo Peralta

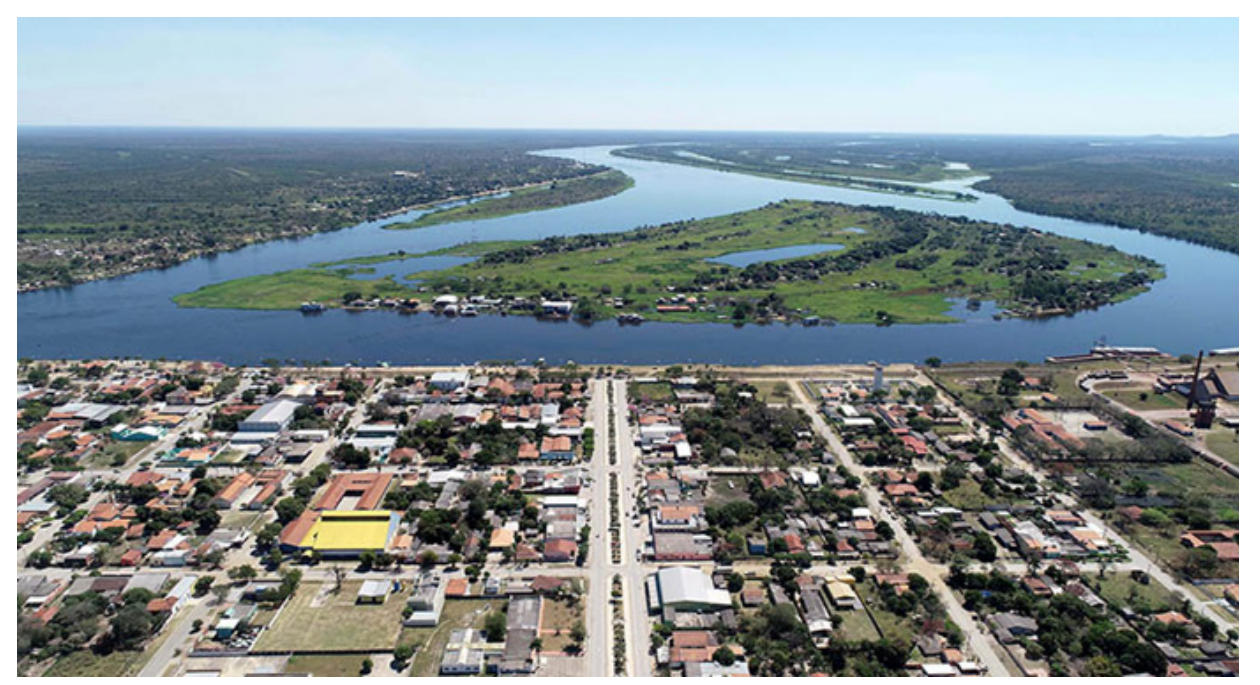

Foto: Porto Murtinho Noticias

Actualmente, en Brasil tomaron volumen las discusiones sobre las "fronteras", no solamente bajo el punto de vista de la demarcación territorial como en el pasado, sino especialmente como preocupación y fuente de análisis de la rutina del hombre de la frontera. Estos debates afectan a dos corrientes de pensamiento, una con una visión negativa, que ve la frontera como una tierra "sin ley", que sirve de corredor para la entrada de drogas, armas y tráfico de personas, entre otros crímenes y otra, con una visión positiva, que ve la frontera como una oportunidad de cooperación y estrechamiento de lazos, de mayores relaciones comerciales y de integración de diferentes culturas. Oliveira (2008, p. 9) afirma: "Es en la frontera donde se hacen evidentes las distinciones y semejanzas entre las normas legales y los hábitos culturales de diferentes países. Allá se burlan con mayor intensidad las reglas sociales tenidas como legítimas."

Es en la frontera donde se anuncian las tendencias de transformación de las "reglas del juego" de las relaciones entre dos países. También son construidas y reconstruidas nuevas rutas de entrada y salida de personas y mercancías, de forma regular o irregular. Sin embargo, por otro lado, es en la frontera también donde hay el entrelazado de culturas, donde ocurre el diálogo entre los países, y donde se construyen nuevas relaciones y culturas, en las que vigoran la solidaridad en las dinámicas de circulación e identificación de las representaciones sobre el otro. Por lo tanto, hay una riqueza infinita de posibilidades en las regiones de frontera.

Brasil posee fronteras con 10 de los 12 países de América del Sur, siendo estos: Bolivia, 
Uruguay, Perú, Venezuela, Colombia, Guyana, Guyana Francesa, Paraguay, Argentina y Surinam. Solamente Chile y Ecuador no poseen frontera con Brasil. De acuerdo con el Censo Demográfico 2010 (IBGE, 2010), el Estado de Mato Grosso del Sur posee 2.4 millones de habitantes distribuidos en 79 municipios. La capital es Campo Grande y el estado es fronterizo con Bolivia y Paraguay y cinco estados brasileños más (San Pablo, Goiás, Mato Grosso, Minas Gerais y Paraná). De los 79 municipios que constituyen el Estado, 44 se encuentran en la región de frontera. Es un local también marcado por la fuerte presencia de pueblos nativos indígenas. Su población está constituida por migrantes de otras regiones de Brasil, que vinieron en busca de nuevas oportunidades a partir de 1977, año en el que ocurrió la división del Estado de Mato Grosso en Mato Grosso y Mato Grosso del Sur. Mato Grosso del Sur, por lo tanto, presenta una característica peculiar, la de la heterogeneidad cultural, que se forma a partir del entrelazado con los territorios de frontera de Bolivia y de Paraguay, ocasionando una gran influencia en los perfiles de las poblaciones locales.

La frontera brasileña con Paraguay se extiende por los estados de Paraná y de Mato Grosso del Sur. Su extensión fronteriza corresponde a 1.365,4 km, de los que 928,5 km son de ríos y 436,9 km por división de aguas (IBGE, 2010). Con Mato Grosso del Sur, Paraguay posee una frontera seca con las siguientes ciudades de Mato Grosso del Sur: Antônio João, Aral Moreira, Bela Vista, Caracol, Coronel Sapucaia, Japorã, Mundo Novo, Paranhos, Ponta Porã, Porto Murtinho y Sete Quedas. Entre esas ciudades, Ponta Porã es la mayor en número de habitantes.

Pensar en la frontera en la actualidad significa considerar también la identidad y principalmente las relaciones de alteridad, que acontecen en las relaciones entabladas con el "yo" y el "otro". Se observa así que ambos construyen su cultura, sus costumbres y sus formas de vida. Además, estas relaciones también se dan en espacios contradictorios, lo que implica directamente la construcción de políticas específicas volcadas hacia esa población y sus demandas más preeminentes.

Es en este contexto que se plantean como pauta urgente las políticas públicas en regiones fronterizas ante la importancia que estas regiones pasan a asumir dentro de estos nuevos contextos, así como el protagonismo de su población. Por cierto, emprendimientos, como el de la Ruta Bioceánica, impactarán toda la región, transformando concepciones y formas de pensar sobre la frontera y sus políticas públicas volcadas hacia las poblaciones locales.

\section{FRONTERA PORTO MURTINHO (BRASIL) X CARMELO PERALTA (PARAGUAY)}

El municipio de Porto Murtinho fue creado en 1911 y emancipado en 1912. La ciudad posee como actividad económica más representativa la agropecuaria, además de actividades como la explotación del quebracho y de la cal de piedra. La ciudad hace frontera con Carmelo Peralta divididos por el Río Paraguay. Esta ubicación le permite la construcción de una identidad bastante peculiar presente en su historia, como, por ejemplo, la participación en conflictos históricos, como la Guerra de la Triple Alianza, y sus influencias provenientes de la frontera paraguaya, como la bebida tradicional llamada "tereré" y otros aspectos de música, danza y culinaria (Ayuntamiento de Porto Murtinho, 2020). Está ubicada en el Pantanal Sur Matogrossense, posee una extensión territorial de $17.735 \mathrm{~km}^{2}$ y está $440 \mathrm{~km}$ distante de la capital, Campo Grande. El local posee atractivos turísticos naturales, con cerros, cascadas y ríos, siendo la pesca uno de los principales atractivos de la región.

De acuerdo con el Instituto Brasileño de Geografía y Estadística (IBGE, 2020) la ciudad

INTERAÇÕES, Campo Grande, MS, v. 22, n. 4, p. xxx-xxx, out./dez. 2021. 
posee una población de 15.372 personas, siendo 65\% residentes del área urbana, una de las menores tasas de MS, y 0,87 de densidad demográfica. Datos recientes del municipio estiman que la población creció hasta 17.298 personas en 2020 y una densidad de 0,98 habitantes por $\mathrm{km}^{2}$. (Ayuntamiento Porto Murtinho, 2020).

En 2010, el Índice de Desarrollo Humano Municipal- IDHM era de 0,666, clasificado como de medio desarrollo, mientras que el IDH de Mato Grosso del Sur $(0,729)$ está considerado como de alto desarrollo debido al agronegocio. El municipio presenta, por lo tanto, un menor desarrollo relativo cuando se le compara a la media del estado, evidenciando la necesidad de una actuación de las políticas públicas para potenciar sus ventajas comparativas.

Una importante consideración a respecto del municipio de Porto Murtinho es que este posee singularidades importantes. Además de ser el segundo mayor municipio de Mato Grosso del Sur en extensión territorial, lo que denota la existencia de una población no solamente urbana sino también importante y diversa población rural, compuesta de indígenas, ribereños, grandes hacenderos y pequeños agricultores. La población es heterogénea en su composición general y gran parte del territorio está ocupada por áreas indígenas alejadas de la región central y es de difícil acceso. El área rural representa buena parte de la extensión territorial del municipio mientras que el área urbana está localizada marcadamente en la franja fronteriza, hecho que genera especificidades en relación con su identidad cultural.

La población indígena se calcula alrededor de más de 1.600- 2.000 habitantes $^{5}$ de las seis aldeas de las etnias Chamacoco, Kadiwéu, Kinikinau y Terena, siendo estas: Aldea Barro Preto (Kadiwéu), Aldea São João (Kadiwéu, Kinikinau), Aldea Tomázia (Kadiwéu), Aldea Alves de Barros (Kadiwéu), Aldea Campina (Kadiwéu) y Aldea Córrego de Ouro (Kadiwéu). Estas se ubican a 280 km de la localización del área urbana, aproximándo de Jardim y Bonito.

A su vez, el municipio de Capitán Carmelo Peralta está ubicado en la región denominada Alto Paraguay, del chaco paraguayo, región aún poco habitada y que tiene un área de $4.798 \mathrm{~km}^{2}$, con una media de 4.432 habitantes. Como Porto Murtinho, Carmelo Peralta también posee una población indígena constituida por la etnia de los ayoreos. Esta está compuesta por diversas comunidades localizadas a las orillas del Río Paraguay. Todas están consideradas como de extrema vulnerabilidad y riesgo social.

El municipio de Carmelo Peralta fue reconoecido como municipio apenas el 2 de mayo de 2008, según la Ley n. 3471 y se encuentra distante de la capital Asunción (730km).

El lado paraguayo también posee la comunidad de la Isla Margarita, que se ubica frente a la ciudad de Porto Murtinho y a la comunidad de Porto Sastre, distante unos $45 \mathrm{~km}$ de la ciudad de Carmelo Peralta. La población tiene su principal fuente de renta y trabajo centrada en las estancias y haciendas locales. La población se dedica a la pesca y a pilotar barcos de travesía entre Porto Murtinho y Carmelo Peralta.

Las dos ciudades destacan en cuanto a la composición de sus poblaciones. Se pueden subrayar las siguientes singularidades:

- Brasileños que se establecieron en territorio paraguayo.

- Personas de origen paraguayo que nacieron en Brasil y residen en Paraguay.

- Indígenas de la etnia Ayoreo que son paraguayos pero registrados efectivamente

5 Hay dificultades de datos en esa población. Estos datos son, por lo tanto no oficiales, tratan de estimativas de la gestión local. 
en suelo brasileño, y que son reconocidos como brasileños residentes en Paraguay.

- Paraguayos que residen de hecho en territorio brasileño y por lo tanto son atendidos también por las políticas públicas brasileñas.

- Brasileños y paraguayos con doble nacionalidad residentes tanto en suelo brasileño como también en suelo paraguayo.

Lo que les une a estos dos municipios, Porto Murtinho y Carmelo Peralta, es la frontera, haciéndolas ciudades consideradas gemelas, de tal forma que para más allá de sus territorios, se identifican sus singularidades e identidades locales, formando una población particularmente delineada por sus culturas y diversidades. Todas esas cuestiones impregnadas por el amplio y nuevo desarrollo regional implican e impactan directamente a las respectivas realidades locales, provocándonos pensar en la importancia de la implementación de políticas públicas consistentes que respondan a las necesidades de los municipios de regiones de frontera.

Las ciudades mencionadas resguardan unas marcadas características en lo que afecta a los diversos tipos de flujo y tránsito transfronteirizo. Estos ocurren siempre por medio de barcos pequeños a motor, cuestión que será diferente tras la construcción del Puente de la Ruta Bioceánica ${ }^{6}$.

Siendo Porto Murtinho un municipio de frontera, una característica marcante es la intensidad de personas que cruzan el río para trabajar en la otra ciudad, tanto la población paraguaya como la población brasileña. Por ejemplo, para la construcción del asfalto perteneciente a la Ruta Bioceánica (2018-2020), los trabajadores paraguayos reclutados entraban por Ponta Porã/MS - Bela Vista/MS (también ciudades fronterizas) y cruzaban el río por Porto Murtinho para llegar a Carmelo Peralta para trabajar en la obra de pavimentación. Ello se debe a la precariedad de la malla de carreteras de Paraguay, cuestión que está siendo solventada con la apertura de una carretera en la región del Chaco Paraguayo.

Por lo tanto, los flujos de trabajadores que viven a un lado de la frontera y trabajan al otro son una peculiaridad bastante común en regiones de frontera. Esta característica está acentuada en esta región y tenderá a presentar un flujo cada vez más intenso en el futuro. Se puede citar que existen personas con familiares residentes al otro lado de la frontera, de modo que tienden a realizar la travesía de forma regular. Además, es importante considerar otra particularidad que tiende a acentuarse con la Ruta Bioceánica, que es el tránsito de camiones de mercancías con cargas de exportación e importación, además de trabajadores de las empresas y de los puertos que están fijando su residencia en la ciudad. Se estima, por lo tanto, en el futuro un flujo intenso de personas que no son originalmente de la región y/o que se encuentran en tránsito.

Estas singularidades determinan la necesidad y la urgencia de proponer acciones concretas y articuladas en pro de la población local, principalmente ribereños e indígenas ayoreos.

\section{CONFIGURACIONES DE LAS POLÍTICAS PÚBLICAS EN LA FRONTERA BRASIL $X$ PARAGUAY}

La expresión "política pública" pasó a ser más utilizada a partir de la década de 1970, ocupando el espacio antes ocupado por la noción de planificación estatal. El término "política" se refiere al conjunto de objetivos que engloba una determinada acción del gobierno y cómo

6 El puente aún será licitado, con una previsión de término para fines de 2023 e inicio de 2024. Tras el establecimiento del puente, las travesías en los pequeños barcos tenderán a disminuir. 
dirigir su ejecución. Por lo tanto, política implica la idea de orientación unitaria para que ciertos fines sean alcanzados, lo que envuelve un conjunto de decisiones gubernamentales que llevarían a concretar los objetivos a través de prácticas programadas y encadenadas de forma coherente (AUGUSTO, 1989).

De este modo, la noción de política pública está relacionada con la intervención estatal en la vida social de la población. El objeto de interferencia estatal está ligado al interés general, aunque lo que pueda ser calificado como público no esté delimitado de forma exenta. De esta forma, aunque las acciones intenten asumir un carácter general y universal, acaban por asumir una forma de jerarquía en el sentido de privilegiar a algunos sujetos en detrimento de otros, conforme a intereses, posiciones y lugares (AUGUSTO, 1989).

En el tema de las políticas públicas de protección social se hace evidente la cuestión de la frontera como problemática específica, ya que en la región hay una existencia de perfiles diferenciados de demanda por parte de la población extranjera habitante del mismo espacio geopolítico (AGUSTINI; NOGUEIRA, 2010).

A partir de la Ruta Bioceánica y su paso por la frontera, rebasando los municipios de Porto Murtinho y Carmelo Peralta, ha sido frecuente cuestionar cuáles serían los impactos sociales y cómo las políticas públicas se deben estructurar en el sentido de garantizar los derechos sociales a su población, lo que, por cierto, se hace pertinente en la medida en que crecen y se modifica las posibilidades dadas a una determinada población bajo el desarrollo económico.

Es justamente en este sentido cuando se hace evidente la importancia de discutir estas cuestiones pues las ciudades de frontera son áreas de rápido y amplio desarrollo socioeconómico y que se modifican rápida y estructuralmente. Las ciudades se ven impactadas significativamente por la forma con la que se expresa la cultura y por los medios de vida de su población. El perfil del trabajador, con sus distintas cualificaciones, frente a los variados cambios esperados de trabajo y empleo y como las familias van a reaccionar a los cambios, ante los deseos y las perspectivas de vida de los jóvenes son presiones que se potencian en una situación de frontera en rápida transformación. De este modo, también hay que adecuar el perfil de las políticas públicas para responder a las necesidades y demandas de las poblaciones que, con toda seguridad, tendrán que alterar también sus metas y perfiles con el fin de alcanzar sus objetivos.

La importancia de estudiar estas cuestiones está en la necesidad de ver el territorio como siendo municipios que aún deben implementar políticas públicas capaces de atender los derechos sociales de las poblaciones, como salud, asistencia social y educación.

Porto Murtinho posee una estructura básica en cuanto a los servicios públicos, considerados adecuados para un municipio de pequeño porte. Si consideramos los equipos en lo que respecta a la salud, asistencia social y educación, se puede afirmar que la ciudad posee medios para prestar una atención satisfactoria. En cuanto a la oferta de equipos públicos, se puede afirmar que Porto Murtinho posee lo suficiente para atender a la población local actual. Sin embargo, con la perspectiva de crecimiento demográfico, en razón del rápido y fuerte incremento de los flujos migratorios, con desplazamientos diarios de personas (camioneros, comerciantes, empresarios y otros), con toda seguridad, el Municipio de Porto Murtinho tendrá que ampliar sus cuadros profesionales e instrumentos de políticas públicas.

En lo que respecta a Carmelo Peralta, debido a su baja densidad de población, se puede citar que esta posee escuela de educación básica y una única unidad de salud básica, pero no posee ningún establecimiento de educación superior y media. Se percibe, por lo tanto, la 
necesidad acuciante de más servicios públicos para asegurar una atención plena a la población, principalmente en lo que respecta a sus derechos educativos.

Con relación a diversos servicios públicos, muchos atendimientos son realizados en Porto Murtinho, debido a la existencia de una estructura básica, principalmente en la atención a la educación y a la salud. Muchos niños paraguayos nacen y son registrados en suelo brasileño y acaban estudiando en las escuelas brasileñas, lo que exige que atraviesen el río todos los días para estudiar. Hay una necesidad de implementar escuelas de enseñanza multicultural, además de implantar más escuelas de enseñanza media y cursos de educación superior.

Con relación a la educación, sabemos hoy de la importancia de las conexiones digitales para la elevación del acceso a la información. Estas son bastante precarias en las dos ciudades. En este sentido, con la implementación de la Ruta Bioceánica, con toda seguridad, serán realizados esfuerzos para tener un avance considerable en esta cuestión y que deberán ser potenciados para la atención a los estudiantes

Con relación a la atención sanitaria, también es acuciante la necesidad de mejorar el servicio en Brasil, pues en casos de tratamiento especializado, el municipio encamina a sus pacientes a Campo Grande, distante $443 \mathrm{Km}$. Algunos de estos tratamientos ya podrían estar siendo realizados en la propia ciudad evitando los gastos con los desplazamientos constantes.

A su vez, debido a la falta de equipamiento y de médicos en Paraguay, los servicios más especializados en salud solo se encuentran en Asunción, en la capital del país. En toda y cualquier intervención que exija una internación o una atención de mayor complejidad, se hace necesario buscar un hospital distante de la ciudad pues en Carmelo Peralta solo existe una Unidad de Salud Básica, para la atención primaria a la población.

Como en Porto Murtinho hay un hospital, la población de Carmelo Peralta escoge el camino más corto, o sea, entra a Brasil cruzando el río para la atención de emergencia sanitaria. En los casos más graves, el paciente es encaminado a Ponta Porã y vuelve a ingresar en Paraguay por Pedro Juan Caballero llegando a Asunción ${ }^{7}$, donde tiene acceso a la atención médico-hospitalar. Ello se da debido a la precariedad de la infraestructura física del Chaco Paraguayo, lo que deberá ser solventado con la pavimentación de la carretera entre Carmelo Peralta y la ciudad de Pozo Hondo, en la frontera con Argentina.

Por lo tanto, para fines de acceso a la atención médico-hospitalar, la ciudade paraguaya posee un acuerdo Paraguay-Brasil para la manutención de un vehículo ambulancia que está en la ciudad de Porto Murtinho, estacionada en el Ayuntamiento, para la conducción de pacientes paraguayos, cuando existe la necesidad, con destino a la capital Asunción, cruzando el territorio brasileño. Así, en casos de una situación de emergencia, un paciente residente en Carmelo Peralta recibe un primer atendimiento en el Hospital de Porto Murtinho pero, conforme sea su situación de salud, puede ser posteriormente encaminado para su atención en Asunción, si tiene condiciones de salud para realizar el desplazamiento. Situaciones como esta son comunes en regiones de frontera lo que subraya la importancia del diálogo constante entre los países.

Es evidente la necesidad de establecer mayores acuerdos bilaterales o mecanismos definidos por las dos ciudades para que toda la población pueda ser atendida de forma rápida y eficiente y no de la forma actual, aún precaria y emergencial, motivada más por razones humanitarias que por el efectivo deseo de prestar una asistencia de salud de calidad para todos.

7 El recorrido Porto Murtinho a Asunción es de $647.7 \mathrm{Km}$.

INTERAÇÕES, Campo Grande, MS, v. 22, n. 4, p. xxx-xxx, out./dez. 2021. 
Es importante resaltar que estos son apenas algunos ejemplos de situaciones vividas en las ciudades de Porto Murtinho/Brasil X Carmelo Peralta/Paraguay que atestiguan las singularidades de la región de frontera y que demuestran la importancia de dar una atención especial a las políticas y potencialidades de la región de frontera.

\section{POSIBILIDADES Y ARTICULACIONES BILATERALES ENTRE PAÍSES EN EL CAMPO DE LAS POLÍTICAS PÚBLICAS}

Al estudiar las cuestiones que envuelven el territorio Porto Murtinho/Carmelo Peralta, partimos de la realidad local y de las especificidades de la región para comprender las cuestiones relacionadas a la implementación de la Ruta Bioceánica, hoy una realidad. Por lo tanto, la implementación de 06 puertos locales para la salida de granos, instalación de nuevas empresas, la expansión del turismo local, y principalmente la apertura de una conexión de paso rápido y seguro entre las dos orillas del río Paraguay, por cierto, demandan una reconfiguración total de la ciudad y de la región. Así, si, por un lado, la expansión traerá la implementación de nuevos empleos y la articulación regional con los países vecinos, de otro, se acentuarán las desigualdades ya vigentes en la región, de forma que se hace cada vez más necesaria la construcción de políticas capaces de minimizar y prevenir esas cuestiones.

La primera de ellas es la cuestión del trabajo. Si, por un lado, se sabe que hoy los jóvenes y personas con edad activa para el mercado de trabajo en las dos ciudades no poseen una capacitación profesional para las nuevas plazas de empleo que probablemente surgirán, también podrán beneficiarse por permanecer en sus respectivas ciudades de origen siempre y cuando sean elaboradas políticas que promuevan formas de capacitación para la población local de modo que se atiendan las nuevas demandas que están surgiendo en las ciudades.

Además, lo que se ha visto es justamente lo contrario. Un ejemplo está en la construcción de la pavimentación en el Paraguay. La empresa responsable del trabajo contrató solamente paraguayos, de acuerdo con las cláusulas inseridas en la contratación de este servicio. Sin embargo, no consiguió mano de obra local suficiente para la realización de la obra, teniendo que contratar paraguayos provenientes de otras regiones. Otro ejemplo, podría ser dado en la medida en que se instalan nuevos puertos y no se disponga de mano de obra local para suplir las necesidades de estas empresas. Hay que desarrollar programas locales de capacitación tanto para paraguayos como brasileños, de modo que se suplan futuramente las distintas demandas.

Una buena práctica sería la articulación entre empresas locales en lo que respesta a la contratación de las poblaciones de los dos territorios, favoreciendo el empleo y la renta para ambas ciudades, conforme vayan siendo implementados nuevos emprendimientos. En la misma dirección sería la implementación de cursos de cualificación y profesionalizantes para ambas ciudades. Estas podrían ser iniciativas conjuntas resultantes de un amplio diálogo entre los países con base en el reconocimiento de las demandas locales y desde la perspectiva de una integración más amplia y profunda.

De acuerdo con la posibilidad de la integración se espera que las poblaciones puedan elevar su nivel de renta y disminuir las desigualdades vigentes, obteniendo acceso a nuevas ofertas de empleo, promoviendo una mayor movilidad social y ascensión profesional y permitiendo descubrir nuevos talentos hoy aún ocultos, principalmente en lo que respecta a los más jóvenes dándoles posibilidades de escapar a tantas vulnerabilidades existentes en estos territorios. 
Otros puntos importantes son la necesidad de disponer de mecanismos de políticas públicas que respondan a las nuevas demandas, como ejemplo, escuelas de enseñanza media multiculturales. Además, la enseñanza de lenguas debería ser incentivada, como el aprendizaje del portugués para los niños de la frontera paraguaya y del español para los niños residentes en el lado brasileño. El abordaje multicultural debe ser promovido a lo largo de la Ruta.

Respecto a las políticas de asistencia social y salud, la importancia de la construcción de nuevos instrumentos para suplir las demandas y formar profesionales cualificados con una política de permanencia evitando la rotatividad de los mismos del local. En este sentido, creemos que el potencial de la Ruta puede promover la creación de nuevos empleos en la región fijando a los profesionales en el local y evitando que los jóvenes se desplacen a otros centros urbanos.

Otra acción importante es sobre las cuestiones de uso de documentación irregular y las vías de posibles regularizaciones, o sea, de brasileños que residen en Paraguay o de paraguayos residentes en Brasil y que, por ventura, no poseen documentos de residencia. En estos casos, los acuerdos comunes pueden colaborar para la resolución de esas dificultades de forma rápida y segura. Protocolos internacionales y la prestación de servicios bilaterales entre ciudades gemelas son, por lo tanto, considerados de extrema importancia y pueden solucionar problemáticas comunes, proporcionando una convivencia sana entre los individuos y la materialización de los derechos sociales y humanos, reduciendo, así, las desigualdades tan difusamente encontradas en esos territorios.

\section{CONSIDERACIONES FINALES}

La Ruta Bioceánica llega a los municipios de Porto Murtinho y Carmelo Peralta con la promesa de traer consigo un desarrollo económico y social, de modo que la prospección nos indica que la región puede atraer cada vez más inversores, emprendimientos de pequeño, medio y gran porte para la región, aumentando aún más el flujo de personas entre los países, así como la población local de ambos lados de la frontera. Pero para que las potencialidades puedan realmente contribuir a mejorar las condiciones de vida de la población, deben ser planeadas con el propósito de mejorar la calidad de vida de los individuos. De este modo, será posible integrar la región y proponer prácticas de políticas públicas locales sensibles a las transformaciones en curso y, principalmente favorecer una mayor autonomía local y menos centralismo político para la región.

Así, las políticas públicas deben estar atentas y preparadas para el aumento significativo de usuarios de servicios, así como debe garantizar derechos sociales y distribución de renta por medio de programas y acciones volcadas hacia las especificidades de un local en desarrollo. Es decir, programas profesionalizantes y de incentivo educacional deben ser elaborados para que las oportunidades de empleo sean atendidas por la población local, haciendo que la renta permanezca en la ciudad y no sea necesario que personas de fuera vengan a ocupar plazas que exigen una mayor cualificación. La creación de nuevos empleos locales podrá motivar a los jóvenes a trabajar en su ciudad de origen y no mudarse a otros centros urbanos.

Acciones específicas que consideren las subjetividades de la región y la actuación en conjunto hace que ambos pueblos crezcan, que acuerdos comerciales sean posibles, y que derechos sociales sean garantizados. Pero es necesario que las relaciones entre los países y sus fronteras dejen de responder a una lógica de la competición y de la exclusión y pasen a orientarse 
por una lógica de cooperación y de colaboración.

El desarrollo económico que ocurrirá podrá alcanzar a su población y para ello, ella también debe estar envuelta en los procesos de decisión y planificación de políticas, para que las demandas reales y cotidianas sean atendidas.

Además, las particularidades de la configuración geográfica de Porto Murtinho deben ser consideradas, dada su gran extensión territorial y diversidad en el perfil de su población (urbana, fronteriza, indígenas y rural), características que demandan políticas que consigan atender a todos. Mucho más que atender a la población local que tiende a crecer, este es un municipio caracterizado esencialmente por ser fronterizo, es decir, su rutina, su pueblo, su cultura, sus creencias y costumbres superaban la cuestión de la frontera resultando en una identidad local subjetiva que debe ser valorada en su singularidad.

De este modo, es importante que se propicie el fortalecimiento del conocimiento recíproco reforzando la identidad local como potenciadora que deberá fortalecer un sentimiento generalizado de solidaridad y pertenencia de una base geográfica común y que, a su vez, reflejará un reconocimiento de la relevancia de la integración y de sus instrumentos normativos.

Finalmente, el establecimiento de una relación dialogante entre los países de la ruta por la cual se valoren las regionalidades es el primer paso para el éxito de cualquier emprendimiento. Este camino tendrá crédito si se implementan acciones y programas donde las poblaciones locales puedan estar involucradas en los procesos decisorios de lo que quieren para sí y para sus ciudades. Una visión valorativa para las diversidades locales, para la cuestión ambiental, para las poblaciones debe ser imprescindible para cualquier planeamiento del desarrollo regional soñado por los países que constituyen la Ruta Bioceánica, promoviendo una integración capaz de superar las fronteras, pero por encima de todo imprimiendo respeto y potenciando las singularidades de cada cual. De este modo, las fronteras dejan de ser vistas como muros y pasan entonces a ser consideradas como puentes, siendo valoradas en sus potencialidades, identidades y en sus diálogos transfronterizos. Este desafío debe ser abrazado por los gobiernos locales como una ventana de oportunidades y un espacio político y social de valoración de sus respectivas poblaciones.

\section{REFERENCIAS}

AGUSTINI, Josiane; NOGUEIRA, Vera Maria Ribeiro. A descentralização da política nacional de saúde nos sistemas municipais na linha da fronteira do Mercosul. Serviço Social \& Sociedade, n. 102, p. 222-243, 2010. AUGUSTO, Maria Helena Oliva. Políticas públicas, políticas sociais e política de saúde: algumas questões para reflexão e debate. Tempo Social; Rev. Sociol. USP, 1(2): 105-119, 1989.

IBGE. Instituto Brasileiro de Geografia e Estatística, 2018. Disponível em <https://sidra.ibge.gov.br/ tabela/5938\#notas-tabela> Acesso em 20 de novembro de 2020.

IBGE, Instituto Brasileiro de Geografia e Estatística. (2010) Cidades e Estados. Mato Grosso do Sul. Disponível em <https://www.ibge.gov.br/cidades-e-estados/ms.html> Acesso em 11 de novembro de 2020. KLEINSCHMITT, Sandra Cristiana; AZEVEDO, Paulo Roberto; CARDIN, Eric Gustavo. A tríplice fronteira internacional entre Brasil, Paraguai e Argentina: contexto histórico, econômico e social de um espaço conhecido pela violência e pelas práticas ilegais. Perspectiva geográfica, v. 8, n. 9, 2013.

OLIVEIRA, Tito Carlos Machado de. América Platina: educação, integração e desenvolvimento territorial. Campo Grande, MS: Ed. UFMS, 2008.

PREFEITURA DE PORTO MURTINHO. História da Cidade. 2020. Disponível em <http://www.portomurtinho. ms.gov.br/historia> Acesso em 03 de março de 2020.

SOUZA, E. B. C. Tríplice fronteira: fluxos da região Oeste do Paraná com o Paraguai e Argentina. Terr@ 
Plural, v. 3, n. 1, p. 103-116, 2009.

\section{Sobre as autoras e o autor:}

Luciane Pinho de Almeida: Possui graduação em Serviço Social (1989) e Pedagogia (1996) pela Universidade Católica Dom Bosco- UCDB, Mestrado (2000) e Doutorado (2004) em Serviço Social pela Universidade Paulista Júlio de Mesquita Filho UNESP- SP. Está na Universidade Católica Dom Bosco desde 1990, onde ocupou os cargos de Diretora de Assuntos Comunitários (2007-2009) e Pró-Reitora de Extensão e Assuntos Comunitários (2009-2018). É líder do Grupo de Estudos e Pesquisas em Teoria Sócio-Histórica, Migrações e Políticas Sociais- GEPEMPS e do Laboratório de Estudos Psicossociais em saúde frente à contextos de desigualdade social- LEPDS, cadastrados no CNPQ. E-mail: Ipinhoa@hotmail.com. ORCID: http://orcid.org/0000-0002-7003-9264

Gabriela Pereira da Silva: Mestranda, bolsista CAPES, em Psicologia com área de concentração em Psicologia da Saúde pela Universidade Católica Dom Bosco. Graduada em Psicologia pela Universidade Católica Dom Bosco. Participa do Grupo de Estudos e Pesquisas em Teoria SócioHistórica, Migrações e Políticas Sociais (GEPEMPS) e do Laboratório de Pesquisas Psicossociais em Saúde frente a Contextos de Desigualdade Social do Programa de Mestrado e Doutorado em Psicologia da Universidade Católica Dom Bosco, sob a coordenação da Profa. Dra. Luciane Pinho de Almeida. E-mail: gabipereira_18@hotmail.com. ORCID: https://orcid.org/0000-0003-2940-1003

Gabriel de Souza Sales: Acadêmico de Psicologia pela Universidade Católica Dom Bosco (UCDB). Participa do Grupo de Estudos e Pesquisas em Teoria Sócio- Histórica, Migrações e Políticas Sociais do Programa de Mestrado e Doutorado em Psicologia da Universidade Católica Dom Bosco, sob a coordenação da Profa. Dra Luciane Pinho de Almeida. Participa do Programa Institucional de Bolsas de Iniciação Científica (PIBIC), no projeto de pesquisa intitulado Migrações, saúde e Assistência Social: Discutindo políticas de Assistência Social e saúde no contexto migratório com o Plano de Trabalho: Migração, saúde e assistência social: discutindo a empregabilidade para migrantes residentes no Estado de Mato Grosso do Sul, coordenado pela Profa. Dra. Luciane Pinho de Almeida.E-mail: gabriel08sales@hotmail.com. ORCID: https://orcid.org/0000-0003-3979-9692.

Livia Elena Cunha Laura: Acadêmica de Psicologia, voluntária PIBIC do Projeto Rede Intersetorial de Políticas Públicas: Políticas de saúde na fronteira de Porto Murtinho/MS, financiado pela FUNDECT, bolsista do Projeto de Extensão Cidadania Sem Fronteiras e membro do Grupo de Estudos e Pesquisas em Teoria Sócio - Histórica, Migração e Políticas Sociais (GEPEMPS), coordenado pela Prof. Dra. Luciane de Pinho Almeida, em Universidade Católica Dom Bosco (UCDB). E-mail: liviaelenalaura@gmail.com. ORCID: https://orcid.org/0000-0002-0714-3341 